\title{
Belgeo
}

Revue belge de géographie

$4 \mid 2019$

Les politiques de mobilité urbaine en Europe après la crise : adaptations et innovations

\section{Editorial : Les politiques de mobilité après la crise : adaptations et innovations}

\section{Nacima Baron}

\section{(2) OpenEdition}

Journals

Édition électronique

URL : http://journals.openedition.org/belgeo/36695

DOI : $10.4000 /$ belgeo.36695

ISSN : 2294-9135

Éditeur :

National Committee of Geography of Belgium, Société Royale Belge de Géographie

Référence électronique

Nacima Baron, «Editorial : Les politiques de mobilité après la crise : adaptations et innovations »,

Belgeo [En ligne], 4 | 2019, mis en ligne le 12 février 2020, consulté le 24 septembre 2020. URL : http:// journals.openedition.org/belgeo/36695; DOI : https://doi.org/10.4000/belgeo.36695

Ce document a été généré automatiquement le 24 septembre 2020.

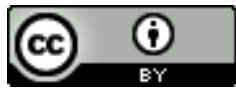

Belgeo est mis à disposition selon les termes de la licence Creative Commons Attribution 4.0 International. 


\title{
Editorial : Les politiques de mobilité après la crise : adaptations et innovations
}

\author{
Nacima Baron
}

1 Les hommes politiques somment le système de mobilité, façonné à l'époque moderne par les révolutions énergétique et industrielle, de se réformer. Pas une journée sans qu'ils n'évoquent la nocivité de l'automobile, la congestion des transports collectifs, les bienfaits de la marche ou du vélo ... Leurs discours abordent toute une série de menaces, dont l'«ubérisation» et, par-delà, l'émergence d'un techno-pouvoir néolibéral fondé sur la donnée et la surveillance, ou bien ils abordent une collection d'opportunités, quand ils associent par le mot et l'image mobilité décarbonée et urbanité apaisée. Ces sujets sont mis à l'agenda à partir d'une évidence, tant la mobilité est une préoccupation omniprésente dans la vie quotidienne des citadins, mais aussi à partir d'argumentaires plus implicites: la mobilité obsède le politique parce que la transformation des manières de bouger est sans doute une métaphore de la Transition, entendue comme mise en mouvement des consciences et des énergies.

2 Au-delà d'une proposition d'analyse (qui serait forcément incomplète) de l'évolution des politiques de mobilité, ce numéro rapproche deux à deux des couples de termes. Le premier couple associe les transformations de l'action publique et les évolutions de la mobilité comme objet social, économique et technique; le second couple de notions conjugue non pas la crise, mais les crises, au sens des innombrables ruptures que nos sociétés d'Europe du Nord-Ouest ont traversées depuis 2008 avec les germes d'innovation et de transformation qu'elles engendrent. Ainsi, d'un côté, les auteurs de ce numéro ont cherché à éclairer les raisons pour lesquelles l'action publique se saisit de manière encore plus volontariste qu'avant des questions de mobilité des voyageurs et ils ont interrogé d'un autre côté l'émergence de stratégies de mobilité en tant qu'elles produisent de nouvelles ressources politiques. Pour ce faire, ils proposent de réarticuler l'évolution des politiques de mobilité avec un référentiel central (mais ambigu), celui de l'écologisation progressive des politiques publiques. On entend par là 
le fait que les élus, de manière très transpartisane, prennent en compte plus sérieusement qu'avant les externalités négatives du système automobile fondé sur les carburants fossiles. Plus globalement, ils interrogent les articulations des mobilités avec d'autres champs de l'action publique (le social, par exemple) et les réinscrivent dans des récits d'action publique et des cultures du déplacement en évolution. A partir de cet assemblage, quatre entrées permettent d'introduire les résultats des sept recherches présentées dans le dossier.

\section{Crise et mobilité, crise de la mobilité}

Ce numéro ne tente pas de répondre à la question de savoir si - et comment - la crise de 2008 a affecté les modèles dominants de mobilité. Il essaye plutôt d'articuler des idées associant un possible tournant des politiques de mobilité, des évolutions respectives de l'économique, du social et des systèmes de décision publique qui ont produit des effets variables, parfois opposés dans tel ou tel contexte territorial. Ainsi la dégradation des finances publiques de l'Etat a causé l'abandon de nombreux projets qualifiés de "grands" donc chers, comme des autoroutes et des lignes à grande vitesse, mais il apparait aussi que la crise a été un excellent argument pour légitimer une relance keynésienne fondée sur l'investissement dans l'infrastructure, comme c'est évoqué dans l'article de Faure. Ailleurs, la diminution conjoncturelle de la fréquentation des transports collectifs en période de chômage fort a été facteur de déséquilibre des comptes d'exploitation des transporteurs mais aussi a occasionné la relance de débats locaux qui ont abouti à des expériences de gratuité complète du service, l'acteur public prenant à sa charge les coûts d'exploitation. Enfin, la crise renvoie à des arbitrages en termes de pouvoir d'achat des ménages, puisque la hausse du coût de l'essence induit une modification des pratiques de déplacement, mais cette perte de pouvoir d'achat entraine aussi des capacités d'investissement plus contraintes dans le renouvellement du parc automobile privé et suscite donc l'émergence de politiques publiques d'encouragement à l'achat d'autres types de moyens de transport (par exemple des subventions à l'acquisition de vélos à assistance électrique). Ainsi, la contraction économique des moyens des acteurs privés, des collectivités publiques et des ménages a non seulement occasionné le freinage, la relance ou la réorientation de politiques de mobilité, mais son inscription dans des cadres idéologiques, normatifs et discursifs renouvelés.

Le numéro questionne donc la fabrique contemporaine des politiques de mobilité dans le contexte des mutations majeures de la dernière décennie, avec la financiarisation de l'économie, l'irruption du digital, la métropolisation et l'accentuation de la fracture socio-spatiale entre centres des grandes villes et périphéries. Les auteurs, chacun à leur manière, tentent de comprendre comment ces processus bousculent violemment le cadre démocratique, institutionnel, organisationnel et opérationnel des déplacements de voyageurs. Ils tentent aussi de préciser l'articulation des temporalités et des échelles d'action du politique. Leur travail confirme que, depuis trois ou quatre décennies, les grands paradigmes hérités de cette politique s'érodent (primat du fonctionnalisme des transports et surdimensionnement des infrastructures, poids de l'Etat central dans la planification, fascination de la vitesse et course en avant technologique....). Leur perspective pour la période plus récente, marquée par la crise, les conduit à conjuguer la chronologie législative et réglementaire (pour la France, du Grenelle de 
l'environnement de 2007 à la Loi d'Orientation sur les Mobilités de 2019) avec des mouvements de recomposition plus fondamentaux des équilibres institutionnels et des phases de la vie démocratique. Ils mettent donc en avant la montée en puissance de l'intercommunalité et des pouvoirs métropolitains, et, pour bien interpréter les politiques locales, la chronologie des victoires électorales et l'organisation d'alternances entre équipes et projets urbains.

\section{Dynamiques circulatoires et territorialisation des modèles des politiques mobilitaires}

5 Ce numéro accorde donc une large place à des cas étudiés en France et en Espagne et il privilégie des entrées locales, de la ville moyenne comme Périgueux (explorée par Roudier) à la grande ville comme Saragosse (analysée par Pueyo et Campos), et à la politique métropolitaine, introduite par Richer et Rabaud dans le cas de Lille. Le choix de ces deux pays n'est pas anodin. L'Espagne, durement touchée par la crise, a dû donner un coup d'arrêt aux chantiers d'infrastructures de transport et a traversé une série de crises immobilières, bancaires, budgétaires, et finalement politiques, avec l'avènement de mairies du changement qui ont opéré des réorientations majeures dans la définition des priorités en matière de mobilité. L'Espagne illustre alors, en contrepoint de la France, un processus de rattrapage accéléré dans l'élaboration d'outils normatifs et dans la mobilisation des discours et de récits consacrés aux mobilités à travers une série d'objets, voire de fétiches (le vélo par exemple). La concentration des travaux sur ces deux pays donne donc à observer des trajectoires et des modèles de circulation et d'appropriation des politiques de mobilité qui contredisent l'idée d'un transfert de modèles de mobilité d'Europe du nord vers le sud mais peuvent ouvrir à une discussion ultérieure sur la circulation de politiques de mobilité dans des réseaux plus circulaires qu'unidirectionnels. Ce prisme latin (élargi à l'Amérique latine avec l'article de Cabrera et Cebollada) permet donc de réfléchir aux conditions d' "atterrissage » et de mise en œuvre des politiques de mobilité et aux opérateurs de ces circulations, experts mais aussi élus ou représentants d'associations qui connectent des discours, des pratiques, des savoirs sur la bonne mobilité du local au global.

6 Dans ces réseaux qui connectent le local à l'international et soutiennent une refonte des politiques de mobilité, un objet concentre en général l'attention des édiles : l'espace public, sa définition, sa qualification, sa valorisation, sa sécurisation, son partage entre modes et usagers. Le numéro y accorde une place en montrant comment durant la crise et depuis, les environnements urbains se transforment très rapidement par extension, rétraction, mutation de l'organisation matérielle des rues et des places, des chaussées, des pistes cyclables ou des trottoirs, qui sont désormais autant des infrastructures de mobilité que des lieux habités et des terrains d'urbanité à part entière. Pour autant, le numéro montre aussi que la réplication des bonnes pratiques et son application dans des contextes locaux variés a induit des débats locaux animés: le programme de mobilité induit ou accompagne un mouvement d'ensemble vers la régénération urbaine (montée des valeurs immobilières), mais cette politique renforce également la fragmentation socio-spatiale des villes. Ainsi, l'utilisation de plans de mobilité a pour effet que certains centre-villes améliorent leurs conditions environnementales, de santé et de qualité de vie tout en déplaçant les effets pernicieux vers certaines 
périphéries. Dessiner la topologie des réseaux d'acteurs publics qui portent des programmes de mobilité et esquisser la géographie des effets des politiques de mobilité appelle les figures du flux et du puzzle. Le flux renvoie à la mobilité des politiques de mobilités, et à la circulation des doctrines et des pratiques de gouvernance des mobilités avec ses processus de diffusion et à partir de foyers porteurs d'innovations dans la définition et la manière de porter les politiques de mobilité. Le puzzle montre plutôt comment ces processus locaux de politisation de la mobilité redessinent des systèmes spatiaux faits de poches spatiales d'innovation et de zones moins actives, cette territorialité pouvant croiser ou recouper des types de lieux (ville versus campagne, centre versus périphérie), des périmètres institutionnels et réglementaires (intercommunalités, territoires de solidarité fiscale), des espaces fonctionnels (périmètres de bassins de déplacement,...).

\section{Que font les politiques de mobilité aux cultures mobilitaires ?}

7 La crise financière de 2008 est plus ou moins synchrone avec l'affirmation d'un intérêt des acteurs publics pour accompagner le développement de pratiques collaboratives et solidaires des véhicules automobiles privés alors que la réalité va plutôt dans le sens de l'érosion de la pratique (Delaunay et Baron). Il faut donc se méfier des assimilations rapides entre volontarisme politique et changements de pratiques de déplacement vers des formes plus sobres énergétiquement et économiquement. Si la précarité pousse certains groupes sociaux à rechercher des solutions de déplacement moins chères, les liens entre politiques d'incitation au changement d'habitudes de déplacement et adoption de comportements de mobilité plus vertueux sont évidemment bien plus complexes. Les politiques d'accompagnement vers des mobilités plus durables s'inscrivent aussi, parfois de manière implicite, dans des politiques disciplinaires, induisant plus ou moins explicitement une réforme des conduites et produisant des discours normatifs qui relient des enjeux d'urbanité, de civilité (ralentissement des vitesses en ville, relation entre individus sur la voie publique) et des représentations du civisme et de la démocratie locale (reconstruction d'un projet de ville rassemblant des citoyens engagés autour d'un projet collectif). Les politiques de mobilité s'adressent donc autant à l'individu (usager ou client d'un service de transport) qu'au sujet politique et, en plus, elles désignent des catégories (groupes cibles d'offre de mobilité, comme les ménages non motorisés) comme elles peuvent participer à la construction de collectifs démocratiques qui se positionnent face à d'autres groupes ou collectifs (les automobilistes, les piétons, les cyclistes ...). C'est à la jonction entre reconnaissance de l'existence de pratiques, éventuellement nouvelles (ou plus souvent réinvesties par de nouveaux groupes sociaux comme le vélo dans les centres-villes, de moins en moins apanage des classes populaires) et de l'expression de droits, individuels et collectifs des usagers, que se constituent et se renégocient sans cesse des cultures et des identités relatives aux mobilités.

D'où, en fin de compte, la conviction fondatrice de ce numéro: les résultats des politiques de mobilités sont assez généralement décevants parce qu'on les analyse de manière encore très sectorielle et dans une perspective de performance. Si on les observe du côté des stratégies de cadrage des mobilités, on est conduit vers une perspective plus transervale. Le numéro montre que les décisions locales qui affectent 
les mobilités s'appuient sur les champs de compétence propres des communautés d'agglomération (cas des pôles gare dans les communautés d'agglomération, par exemple), mais cheminent aussi à travers d'autres cadrages, d'autres argumentaires, d'autres visées. Les politiques de mobilité servent sans doute à faciliter les mouvements domicile-travail ou à lutter contre la congestion, mais aussi au développement économique et touristique, à la lutte contre la précarité à travers l'accessibilité à l'emploi, à la prévention sanitaire ou à la politique éducative. Toute cette variété conduit à soutenir qu'il vaut la peine de proposer une démarche d'observation de cas locaux pour saisir des processus d'expression et d'impulsion de transformation des politiques mobilitaires par le bas, afin de saisir en quoi elles complètent ou au contraire érodent des formes de pilotage des politiques de mobilité déterminées dans des cadres établis par l'État ou par le marché. Par exemple, il est vrai que l'action locale peine à trouver les conditions organisationnelles et institutionnelles d'encadrement des innovations de mobilité. Elle a le plus grand mal à produire un cadre normatif et à suivre les avancées technologiques (du côté des véhicules autonomes ou du côté de la captation et valorisation des données de mobilité). Ainsi Pueyo et Campos montrent le vide réglementaire et le retard d'adaptation des réglementations locales alors que les trottinettes ou les vélos en free floating envahissent les rues. Mais ils nous expliquent également comment la course contre la montre se joue non seulement entre pouvoir municipal, armé d'ordonnances d'occupation de la voirie, et sociétés privées, mais aussi et peut-être surtout entre municipalités et État, les premières saisissant ce combat pour affirmer une forme d'émancipation communale à l'égard de la machine réglementaire étatique tout aussi dépassée.

9 L'entrée des politiques de mobilité par le local offre enfin des perspectives de discussion sur les expériences, les appétences, les résistances issues du local dans les évolutions du service public de mobilité. En contrecoup du renforcement des compétences des autorités organisatrices à des échelons pluri-communaux, on assiste, notamment aux franges des métropoles, à la rationalisation des offres de transport public : les réseaux de bus redessinent des bassins, leurs bouts de lignes peu desservis sont transformés en transport à la demande, services de covoiturage ou d'auto-stop organisé... Ceci accompagne un retour d'intérêt pour l'échelon politique de base, la commune; pour la figure politique centrale, le maire; pour une forme de mobilité : la proximité. Mais quelles sont les capacités politiques et les marges de manœuvre du pouvoir local ? Le numéro peut aider à comprendre les conditions d'élaboration de stratégies locales de mobilité, à questionner les capacités de lecture d'un élu à propos des besoins de mobilité de ses concitoyens, ses connaissances et compétences en matière de technologie, la richesse de ses réseaux et relais dans les mondes de l'expertise, de l'entreprise. Tous ces ingrédients de la fabrique des politiques locales de mobilité font que les décisions et les expérimentations n'empruntent pas des voies uniformes et conduisent vers des scenarios variés : fabrique incrémentale d'une offre de transport public élargie à plus de services, plus d'immatériel, plus de solidarité, contrôlée démocratiquement et pilotée depuis une vision de service public ou bien maintien de grands partages (service public versus marché privé, transport collectif versus individuel) qui modifient au fond assez peu, sous couvert de nouveaux services innovants, les systèmes de pouvoir et les rapports de force établis. 


\section{AUTEUR}

NACIMA BARON

Université Gustave Eiffel, Laboratoire Ville Mobilité Transport, nacima.baron@enpc.fr 